\title{
LINEAR NEUTRAL PARTIAL DIFFERENTIAL EQUATIONS: A SEMIGROUP APPROACH
}

\author{
RAINER NAGEL and NGUYEN THIEU HUY
}

\author{
Received 17 September 2002
}

\begin{abstract}
We study linear neutral PDEs of the form $(\partial / \partial t) F u_{t}=B F u_{t}+\Phi u_{t}, t \geq 0 ; u_{0}(t)=$ $\varphi(t), t \leq 0$, where the function $u(\cdot)$ takes values in a Banach space $X$. Under appropriate conditions on the difference operator $F$ and the delay operator $\Phi$, we construct a $C_{0}$-semigroup on $C_{0}\left(\mathbb{R}_{-}, X\right)$ yielding the solutions of the equation.
\end{abstract}

2000 Mathematics Subject Classification: 34K60, 47D06, 34K30, 34G10.

1. Introduction. In this paper, we study linear neutral PDEs of the form

$$
\begin{gathered}
\frac{\partial}{\partial t} F u_{t}=B F u_{t}+\Phi u_{t} \quad \text { for } t \geq 0, \\
u_{0}(t)=\varphi(t) \quad \text { for } t \leq 0
\end{gathered}
$$

Here, $B$ is some linear partial differential operator, while the operators $F$ and $\Phi$ are called difference operator and delay operator, respectively. We refer to Hale [5, 6], Wu [12, Chapter 2.3], Wu and Xia [13], and Adimy and Ezzinbi [1] for concrete examples and propose an abstract treatment of these equations. For that purpose, we choose a Banach space $X$ and consider the solution $u(\cdot)$ as a function from $\mathbb{R}$ to $X$. Then, the corresponding history function is defined as

$$
u_{t}(s):=u(t+s) \quad \forall t \geq 0, s \leq 0 .
$$

Moreover, $B$ is a linear operator on $X$ (representing the partial differential operator), while $F$ and $\Phi$ are linear operators from an $X$-valued function space; for example, $C_{0}\left(\mathbb{R}_{-}, X\right)$ into $X$. More precisely, we make the following assumption throughout the paper.

Assumption 1.1. On the Banach spaces $X$ and $E:=C_{0}\left(\mathbb{R}_{-}, X\right)$, we consider the following operators:

(i) let $(B, D(B))$ be the generator of a strongly continuous semigroup $\left(e^{t B}\right)_{t \geq 0}$ on $X$ satisfying $\left\|e^{t B}\right\| \leq M e^{\omega_{1} t}$ for some constants $M \geq 1$ and $\omega_{1} \in \mathbb{R} ;$

(ii) let the difference operator $F: E \rightarrow X$ and the delay operator $\Phi: E \rightarrow X$ be bounded and linear. 
Under these assumptions, we will solve (1.1) by constructing an appropriate strongly continuous semigroup on the space $E$. This semigroup will be obtained by proving that a certain operator (see Definition 2.3) satisfies the Hille-Yosida conditions as long as we can write the difference operator as $F=\delta_{0}-\Psi$ with $\Psi$ being "small" (see (2.7)). If the delay and difference operators act only on a finite interval $[-r, 0]$, it can be shown that the smallness of $\Psi$ can be replaced by the condition "having no mass in 0" (see Definition 3.1).

In the case of ordinary neutral functional differential equations on finitedimensional spaces $X$, we refer the readers to Hale and Verduyn Lunel [7, Chapter 9], Engel [3], and Kappel and Zhang [9, 10] for results about wellposedness and asymptotic behavior of the solutions as well as the use of the condition "having no mass in 0" (or, "nonatomic at zero," see Remark 3.2). In the case of infinite-dimensional spaces $X$, such a condition appeared in [11] (see also Datko [2]), where the generator property has been shown under dissipativity conditions for ordinary neutral functional differential equations. Hale [5, 6] and $\mathrm{Wu}[12$, Chapter 2.3] assumed $B$ to generate an analytic semigroup and also obtained a semigroup solving (1.1) in a mild sense if $\Psi$ is nonatomic at zero.

2. Neutral semigroups with infinite delay. Under the assumptions from Section 1 , we consider the Banach space $E:=C_{0}\left(\mathbb{R}_{-}, X\right)$ endowed with the supnorm and the operator $\left(G_{m}, D\left(G_{m}\right)\right)$ on $E$, defined by

$$
\begin{aligned}
D\left(G_{m}\right) & :=\left\{f \in E \cap C^{1}\left(\mathbb{R}_{-}, X\right): f^{\prime} \in E\right\}, \\
G_{m} f & :=f^{\prime} \quad \text { for } f \in D\left(G_{m}\right) .
\end{aligned}
$$

We are now looking for various restrictions of this (maximal) operator yielding generators of strongly continuous semigroups. We start with a simple case.

DEFINITION 2.1. On the space $E=C_{0}\left(\mathbb{R}_{-}, X\right)$, we define the operator $T_{B, 0}(t)$ by

$$
\left[T_{B, 0}(t) f\right](s)= \begin{cases}f(s+t), & s+t \leq 0, \\ e^{(t+s) B} f(0), & s+t \geq 0\end{cases}
$$

for $f \in E$ and $t \geq 0$.

Moreover, we define the operator $\left(G_{B, 0}, D\left(G_{B, 0}\right)\right)$ by

$$
\begin{gathered}
D\left(G_{B, 0}\right):=\left\{f \in D\left(G_{m}\right): f(0) \in D(B), f^{\prime} \in E, f^{\prime}(0)=B f(0)\right\}, \\
G_{B, 0} f:=f^{\prime} \quad \text { for } f \in D\left(G_{B, 0}\right) .
\end{gathered}
$$

We then have the following properties of $G_{B, 0}$ and $\left(T_{B, 0}(t)\right)_{t \geq 0}$ which can be proved as in [8, Proposition 2.8]. 
Proposition 2.2. The following assertions hold:

(i) $\left(T_{B, 0}(t)\right)_{t \geq 0}$ is a strongly continuous semigroup on the space $E$ with the generator $\left(G_{B, 0}, D\left(G_{B, 0}\right)\right)$;

(ii) the set $\{\lambda \in \mathbb{C}: \operatorname{Re} \lambda>0, \lambda \in \rho(B)\}$ is contained in $\rho\left(G_{B, 0}\right)$. Moreover, for $\lambda$ in this set, the resolvent is given by

$$
\left[R\left(\lambda, G_{B, 0}\right) f\right](t)=e^{\lambda t} R(\lambda, B) f(0)+\int_{t}^{0} e^{\lambda(t-\xi)} f(\xi) d \xi \quad \text { for } f \in E, t \leq 0
$$

(iii) the semigroup $\left(T_{B, 0}(t)\right)_{t \geq 0}$ satisfies

$$
\left\|T_{B, 0}(t)\right\| \leq M e^{\omega_{2} t}, \quad t \geq 0
$$

with $\omega_{2}:=\max \left\{0, \omega_{1}\right\}$ for the constants $M$ and $\omega_{1}$ appearing in Assumption 1.1.

We now take the delay operator $\Phi$ and the difference operator $F$ (see Assumption 1.1) to define a different restriction of the operator $G_{m}$.

DEFINITION 2.3. The operator $G_{B, F, \Phi}$ is defined by

$$
\begin{aligned}
G_{B, F, \Phi} f & :=f^{\prime} \text { on the domain } \\
D\left(G_{B, F, \Phi}\right) & :=\left\{f \in D\left(G_{m}\right): F f \in D(B), F\left(f^{\prime}\right)=B F f+\Phi f\right\} .
\end{aligned}
$$

Our aim is to find conditions on $F$ such that the operator $G_{B, F, \Phi}$ becomes the generator of a strongly continuous semigroup. To do so, we write $F$ in the form

$$
F f:=f(0)-\Psi f, \quad f \in E,
$$

for some bounded linear operator $\Psi: E \rightarrow X$. The domain of $G_{B, F, \Phi}$ can then be rewritten as

$$
\begin{aligned}
& D\left(G_{B, F, \Phi}\right) \\
& \quad=\left\{f \in D\left(G_{m}\right): f(0)-\Psi f \in D(B), f^{\prime}(0)=B(f(0)-\Psi f)+\Phi f+\Psi f^{\prime}\right\} .
\end{aligned}
$$

It is now our main result that if the operator $\Psi$ is "small," then $G_{B, F, \Phi}$ is densely defined and satisfies the Hille-Yosida estimates; hence, generates a strongly continuous semigroup.

For that purpose and for $\lambda \in \mathbb{C}$ satisfying $\operatorname{Re} \lambda>0$, we define the operator $e_{\lambda}: X \rightarrow E$ by

$$
\left[e_{\lambda} x\right](t):=e^{\lambda t} x \quad \text { for } t \leq 0, x \in X
$$


THEOREM 2.4. Assume that the difference operator $F$ is of the form (2.7) such that $\Psi$ satisfies the condition $\|\Psi\|<1$. Then, the following assertions hold:

(i) $\lambda \in \rho\left(G_{B, F, \Phi}\right)$ for each $\lambda>\omega_{2}+M\|\Phi\| /(1-\|\Psi\|)$ (with the constants $\omega_{2}$ and $M$ as in Proposition 2.2). For such $\lambda$, the resolvent of $G_{B, F, \Phi}$ has the form

$$
\begin{aligned}
R\left(\lambda, G_{B, F, \Phi}\right) f= & e_{\lambda}\left[\Psi R\left(\lambda, G_{B, F, \Phi}\right)+R(\lambda, B)\left(\Phi R\left(\lambda, G_{B, F, \Phi}\right)-\Psi\right)\right] f \\
& +R\left(\lambda, G_{B, 0}\right) f \quad \text { for } f \in E ;
\end{aligned}
$$

(ii) for $L:=(M+M\|\Psi\|) /(1-\|\Psi\|)$ and $\lambda_{0}:=\omega_{2}+M\|\Phi\| /(1-\|\Psi\|)$,

$$
\left\|R\left(\lambda, G_{B, F, \Phi}\right)\right\| \leq \frac{L}{\left(\lambda-\lambda_{0}\right)} \quad \text { for } \lambda>\lambda_{0}
$$

(iii) for $\lambda>\omega_{0}:=\max \left\{2 \lambda_{0}, \omega_{2}+L\|\Phi\|\right\}$ and $P:=3 e[(M+L)\|\Psi\|+2 M+1]$, it follows that

$$
\left\|R\left(\lambda, G_{B, F, \Phi}\right)^{n}\right\| \leq \frac{P}{\left(\lambda-\omega_{0}\right)^{n}} \quad \forall n \in \mathbb{N}
$$

(iv) the operator $G_{B, F, \Phi}$ is densely defined.

Proof. (i) We first observe that for $u, f \in E$ and $\lambda \in \mathbb{C}$, we have that $u \in$ $D\left(G_{m}\right)$ and $\lambda u-G_{m} u=f$ if and only if $u$ and $f$ satisfy

$$
u(t)=e^{\lambda(t-s)} u(s)+\int_{t}^{s} e^{\lambda(t-\xi)} f(\xi) d \xi \text { for } t \leq s \leq 0 .
$$

Note that for $\lambda>\omega_{2}$ and by (2.4),

$$
\begin{aligned}
u(t)= & e^{\lambda t}[\Psi u+R(\lambda, B)(f(0)+\Phi u-\Psi f)] \\
& +\int_{t}^{0} e^{\lambda(t-\xi)} f(\xi) d \xi \quad \text { for } t \leq 0
\end{aligned}
$$

is equivalent to

$$
u=e_{\lambda}(\Psi u+R(\lambda, B) \Phi u)-e_{\lambda} R(\lambda, B) \Psi f+R\left(\lambda, G_{B, 0}\right) f
$$

If, for each $f \in E$ and $\lambda>\lambda_{0}$, the equation (2.15) has a unique solution $u \in E$, then $u(0)=\Psi u+R(\lambda, B)(f(0)+\Phi u-\Psi f)$. This is equivalent to

$$
(\lambda-B)(u(0)-\Psi u)=\left[\left(\lambda-G_{m}\right) u\right](0)+\Phi u-\Psi\left(\lambda-G_{m}\right) u
$$


or

$$
u^{\prime}(0)=B(u(0)-\Psi u)+\Phi u+\Psi u^{\prime}
$$

Hence, by the above observation and the definition of $G_{B, F, \Phi}$, we have that $u \in D\left(G_{B, F, \Phi}\right)$ and $u=R\left(\lambda, G_{B, \Phi, F}\right) f$. Therefore, to prove (i), we have to verify that for $\lambda>\lambda_{0}$ and each $f \in E$, (2.14) has a unique solution $u \in E$.

Let $M_{\lambda}: E \rightarrow E$ be the linear operator defined as

$$
M_{\lambda}:=e_{\lambda}(\Psi+R(\lambda, B) \Phi)
$$

Since $\lambda>\omega_{2}+M\|\Phi\| /(1-\|\Psi\|)$, we have that $M_{\lambda}$ is bounded and satisfies

$$
\left\|M_{\lambda}\right\| \leq\|\Psi\|+\frac{M\|\Phi\|}{\lambda-\omega_{2}}<1
$$

Therefore, the operator $I-M_{\lambda}$ is invertible, and (2.14) has a unique solution $u=\left(I-M_{\lambda}\right)^{-1}\left(R\left(\lambda, G_{B, 0}\right) f-e_{\lambda} R(\lambda, B) \Psi f\right)$. Thus,

$$
R\left(\lambda, G_{B, F, \Phi}\right) f=M_{\lambda} R\left(\lambda, G_{B, F, \Phi}\right) f-e_{\lambda} R(\lambda, B) \Psi f+R\left(\lambda, G_{B, 0}\right) f
$$

and (2.10) follows.

(ii) By the Neumann series $\left(I-M_{\lambda}\right)^{-1}=\sum_{n=0}^{\infty} M_{\lambda}^{n}$, we have that

$$
\begin{aligned}
\left\|R\left(\lambda, G_{B, F, \Phi}\right)\right\| & =\left\|\sum_{n=0}^{\infty} M_{\lambda}^{n}\left(R\left(\lambda, G_{B, 0}\right)-e_{\lambda} R(\lambda, B) \Psi\right)\right\| \\
& \leq\left(\sum_{n=0}^{\infty}\left\|M_{\lambda}^{n}\right\|\right) \frac{M+M\|\Psi\|}{\left(\lambda-\omega_{2}\right)} \\
& \leq \frac{M+M\|\Psi\|}{\left(\lambda-\omega_{2}\right)} \sum_{n=0}^{\infty}\left(\|\Psi\|+\frac{M\|\Phi\|}{\lambda-\omega_{2}}\right)^{n} \\
& =\frac{M+M\|\Psi\|}{(1-\|\Psi\|)\left(\lambda-\omega_{2}-M\|\Phi\| /(1-\|\Psi\|)\right)} \\
& =\frac{L}{\lambda-\lambda_{0}} .
\end{aligned}
$$

(iii) For $\lambda>\lambda_{0}$ and $u:=R\left(\lambda, G_{B, F, \Phi}\right) f$, we have

$$
\begin{aligned}
u(t)= & e^{\lambda t}\left[\Psi R\left(\lambda, G_{B, F, \Phi}\right) f+R(\lambda, B)\left(\Phi R\left(\lambda, G_{B, F, \Phi}\right) f-\Psi f+f(0)\right)\right] \\
& +\int_{t}^{0} e^{\lambda(t-\xi)} f(\xi) d \xi \quad \text { for } t \leq 0
\end{aligned}
$$


We extend $u$ and $f$ to functions on $\mathbb{R}$ by

$$
\begin{aligned}
& \tilde{u}(t):= \begin{cases}u(t) & \text { for } t \leq 0, \\
e^{\lambda t} g(t) & \text { for } t>0,\end{cases} \\
& \tilde{f}(t):= \begin{cases}f(t) & \text { for } t \leq 0, \\
-e^{\lambda t} g^{\prime}(t) & \text { for } t>0,\end{cases}
\end{aligned}
$$

where we take $g(t):=u(0)+\int_{0}^{t} \varphi(\tau) d \tau$ with

$$
\varphi(t):= \begin{cases}6 t\left[t \lambda^{2}-\lambda\right]\left[\lambda u(0)-\frac{1}{2} f(0)\right]+[\lambda t-1] f(0) & \text { for } 0 \leq t \leq \frac{1}{\lambda} \\ 0 & \text { for } t \geq \frac{1}{\lambda} .\end{cases}
$$

Then, $g$ is continuously differentiable with compact support contained in the interval $[0,1 / \lambda]$ satisfying $g(0)=u(0), g^{\prime}(0)=-f(0)$, and

$$
\left\|e^{\lambda t} g^{\prime}(t)\right\| \leq 3 e[(M+L)\|\Psi\|+2 M+1]\|f\|
$$

for $\lambda>\max \left\{2 \lambda_{0}, \omega_{2}+L\|\Phi\|\right\}$ and all $t \in \mathbb{R}_{+}$. Hence, the functions $\tilde{u}$ and $\tilde{f}$ defined by (2.23) belong to the Banach space $C_{0}(\mathbb{R}, X)$ and satisfy

$$
\begin{gathered}
\tilde{u}(t)=e^{\lambda(t-s)} \tilde{u}(s)+\int_{t}^{s} e^{\lambda(t-\xi)} \tilde{f}(\xi) d \xi \text { for } t \leq s, \\
\|\tilde{f}\| \leq 3 e[(M+L)\|\Psi\|+2 M+1]\|f\| .
\end{gathered}
$$

We now look at the left translation semigroup $(\tilde{T}(t))_{t \geq 0}$ on the Banach space $\tilde{E}:=C_{0}(\mathbb{R}, X)$, that is,

$$
(\tilde{T}(t) \tilde{f})(s):=\tilde{f}(s+t) \quad \forall \tilde{f} \in \tilde{E}, s \in \mathbb{R}, t \geq 0 .
$$

This semigroup is strongly continuous on $\tilde{E}$ and its generator is $\tilde{G}_{m}:=d / d s$ on the domain $D\left(\tilde{G}_{m}\right):=\left\{f \in \tilde{E} \cap C^{1}(\mathbb{R}, X): f^{\prime} \in \tilde{E}\right\}$ (see [4, Chapter II.2]). Furthermore, we observe that for $v, w \in \tilde{E}$ and $\lambda \in \mathbb{C}$, we have that $v \in D\left(\tilde{G}_{m}\right)$ and $\lambda v-\tilde{G}_{m} v=w$ if and only if $v$ and $w$ satisfy (2.26). Since $\lambda \in \rho\left(\tilde{G}_{m}\right)$ for $\lambda>\lambda_{0}$, we obtain that $\tilde{u}=R\left(\lambda, \tilde{G}_{m}\right) \tilde{f}$ for $\lambda>\lambda_{0}$, where $\tilde{u}$ and $\tilde{f}$ are defined as in (2.23).

Therefore, by (2.23), we have that

$$
\left[R\left(\lambda, G_{B, F, \Phi}\right) f\right](t)=u(t)=\tilde{u}(t)=\left[R\left(\lambda, \tilde{G}_{m}\right) \tilde{f}\right](t)
$$

for $t \leq 0$ and $\lambda>\max \left\{2 \lambda_{0}, \omega_{2}+L\|\Phi\|\right\}=: \omega_{0}$. 
By induction, we obtain

$$
\left[R\left(\lambda, G_{B, F, \Phi}\right)^{n} f\right](t)=\left[R\left(\lambda, \tilde{G}_{m}\right)^{n} \tilde{f}\right](t) \text { for } t \leq 0, \lambda>\omega_{0} .
$$

Using the fact that $\tilde{G}_{m}$ is the generator of the strongly continuous semigroup $(\tilde{T}(t))_{t \geq 0}$ on $\tilde{E}$, and by inequality (2.27), we have

$$
\begin{aligned}
\left\|\left[R\left(\lambda, G_{B, F, \Phi}\right)^{n} f\right](t)\right\| & =\left\|\left[R\left(\lambda, \tilde{G}_{m}\right)^{n} \tilde{f}\right](t)\right\| \\
& \leq \frac{\|\tilde{f}\|}{\lambda^{n}} \leq \frac{3 e[(M+L)\|\Psi\|+2 M+1]}{\left(\lambda-\omega_{0}\right)^{n}}\|f\|
\end{aligned}
$$

for all $t \leq 0, \lambda>\omega_{0}$, and all $n \in \mathbb{N}$. Therefore, putting $P:=3 e[(M+L)\|\Psi\|+$ $2 M+1]$, we obtain

$$
\left\|R\left(\lambda, G_{B, F, \Phi}\right)^{n} f\right\| \leq \frac{P}{\left(\lambda-\omega_{0}\right)^{n}}\|f\| \quad \text { for } \lambda>\omega_{0}, n \in \mathbb{N} .
$$

(iv) For $\lambda>\lambda_{0}$, we consider the operator $S: E \rightarrow E$ defined by

$$
S f:=-e_{\lambda} R(\lambda, B) \Psi f+R\left(\lambda, G_{B, 0}\right) f, \quad f \in E .
$$

Observe that if its range $I m S$ is dense in $E$, then we have, by (2.20), that $D\left(G_{B, F, \Phi}\right)=R\left(\lambda, G_{B, F, \Phi}\right) E=\left(I-M_{\lambda}\right)^{-1} I m S$ is dense in $E$. Therefore, it is enough to verify that $I m S$ is dense in $E$. Since $D\left(G_{m}\right)$ is dense in $E$, we only need to show that $\overline{\operatorname{ImS}} \supset D\left(G_{m}\right)=D\left(\lambda-G_{m}\right)$.

In fact, for $u \in D\left(\lambda-G_{m}\right)$, there exists $f \in E$ such that

$$
u(t)=e^{\lambda t} u(0)+\int_{t}^{0} e^{\lambda(t-\xi)} f(\xi) d \xi \text { for } t \leq 0
$$

Since the operator $B$ is densely defined, there exists a sequence $\left(y_{n}\right) \subset D(B)$ such that $\lim _{n \rightarrow \infty} y_{n}=u(0)$. Let $\left(x_{n}\right) \subset X$ be a sequence such that $R(\lambda, B) x_{n}=$ $y_{n}$.

For each $n \in \mathbb{N}$, we choose a real-valued, continuous function $\alpha_{n}(t)$ with support contained in $\left[\max \left\{-1 / n,-1 / n\left\|x_{n}\right\|\right\}, 0\right]$ satisfying $\alpha_{n}(0)=1$ and $\sup _{t \leq 0}\left|\alpha_{n}(t)\right| \leq 1$.

By the condition $\|\Psi\|<1$, we have that the functions

$$
f_{n}(\cdot):=\left(I-\alpha_{n}(\cdot) \Psi\right)^{-1}\left(\alpha_{n}(\cdot)\left(x_{n}-f(0)\right)+f(\cdot)\right), \quad n \in \mathbb{N},
$$


belong to $E$. Moreover, these functions satisfy

$$
\begin{gathered}
f_{n}(t)=\alpha_{n}(t)\left(x_{n}+\Psi\left(f_{n}\right)-f(0)\right)+f(t), \\
f_{n}(0)-\Psi\left(f_{n}\right)=x_{n}, \\
\left\|f_{n}\right\| \leq \frac{\left\|x_{n}\right\|+2\|f\|}{1-\|\Psi\|} .
\end{gathered}
$$

We now put

$$
u_{n}(t):=e^{\lambda t} R(\lambda, B)\left(f_{n}(0)-\Psi f_{n}\right)+\int_{t}^{0} e^{\lambda(t-\xi)} f_{n}(\xi) d \xi
$$

Then, $u_{n}=S f_{n}$; hence $u_{n} \in \operatorname{Im} S$, and for $\lambda>\lambda_{0}$, we obtain

$$
\begin{aligned}
& \left\|u_{n}(t)-u(t)\right\| \\
& =\left\|e^{\lambda t}\left(R(\lambda, B) x_{n}-u(0)\right)+\int_{t}^{0} e^{\lambda(t-\xi)}\left(f_{n}(\xi)-f(\xi)\right) d \xi\right\| \\
& \leq\left\|y_{n}-u(0)\right\|+\int_{t}^{0}\left|\alpha_{n}(t)\right|\left(\left\|x_{n}\right\|+\|\Psi\|\left\|f_{n}\right\|+\|f(0)\|\right) d t \\
& \quad \leq\left\|y_{n}-u(0)\right\|+\int_{\max \left\{-1 / n,-1 / n\left\|x_{n}\right\|\right\}}^{0}\left(\left\|x_{n}\right\|+\frac{\|\Psi\|\left(\left\|x_{n}\right\|+2\|f\|\right)}{1-\|\Psi\|}+\|f\|\right) d t \\
& \leq\left\|y_{n}-u(0)\right\|+\frac{1}{n(1-\|\Psi\|)}+\frac{(1+\|\Psi\|)\|f\|}{n(1-\|\Psi\|)} \stackrel{n \rightarrow \infty}{\longrightarrow} 0 \text { uniformly } \forall t \in \mathbb{R}_{-} .
\end{aligned}
$$

This means that $\lim _{n \rightarrow \infty} u_{n}=u$. Thus, ImS is dense in $E$.

The Hille-Yosida theorem now yields the following corollary.

COROLLARY 2.5. Let the difference operator $F$ have the form (2.7) with $\Psi$ satisfying $\|\Psi\|<1$. Then the operator $G_{B, F, \Phi}$ generates a strongly continuous semigroup $\left(T_{B, F, \Phi}(t)\right)_{t \geq 0}$ on $E$ satisfying

$$
\left\|T_{B, F, \Phi}(t)\right\| \leq P e^{\omega_{0} t}, \quad t \geq 0
$$

where the constants $P$ and $\omega_{0}$ are defined as in Theorem 2.4.

We now conclude this section by a corollary about well-posedness of (1.1). To this end, we denote by $t \mapsto u_{t}(\cdot, \varphi)$ the classical solution of (1.1) corresponding to the initial condition $u_{0}=\varphi$, that is, $t \mapsto u_{t}(\cdot, \varphi)$ is continuously differentiable and satisfies (1.1). 
COROLlaRY 2.6. Assume that the difference operator $F$ is of the form (2.7) such that $\Psi$ satisfies $\|\Psi\|<1$. Then, (1.1) is well posed. More precisely, for every $\varphi \in D\left(G_{B, F, \Phi}\right)$, there exists a unique classical solution $u_{t}(\cdot, \varphi)$ of $(1.1)$ given by

$$
u_{t}(\cdot, \varphi)=T_{B, F, \Phi}(t) \varphi
$$

and for every sequence $\left(\varphi_{n}\right)_{n \in \mathbb{N}} \subset D\left(G_{B, F, \Phi}\right)$ satisfying $\lim _{n \rightarrow \infty} \varphi_{n}=0$,

$$
\lim _{n \rightarrow \infty} u_{t}\left(\cdot, \varphi_{n}\right)=0
$$

uniformly in compact intervals.

Proof. By Corollary 2.5, the operator $\left(G_{B, F, \Phi}, D\left(G_{B, F, \Phi}\right)\right)$ defined by $(2.6)$ is the generator of the strongly continuous semigroup $\left(T_{B, F, \Phi}(t)\right)_{t \geq 0}$.

For $\varphi \in D\left(G_{B, F, \Phi}\right)$, we put $u_{t}:=T_{B, F, \Phi}(t) \varphi$. Then, it is clear that $u_{t} \in$ $D\left(G_{B, F, \Phi}\right) \subset D\left(G_{m}\right)$. We now show that $u_{t}$ satisfies (1.1). Indeed, we have

$$
\begin{aligned}
\frac{d}{d t} F u_{t} & =\lim _{h \rightarrow 0} \frac{F u_{t+h}-F u_{t}}{h}=\lim _{h \rightarrow 0} \frac{F T(t+h) \varphi-F T(t) \varphi}{h} \\
& =F \lim _{h \rightarrow 0} \frac{T(h) T(t) \varphi-T(t) \varphi}{h}=F G_{B, F, \Phi} T(t) \varphi \\
& =B F T(t) \varphi+\Phi T(t) \varphi=B F u_{t}+\Phi u_{t} .
\end{aligned}
$$

For the uniqueness of the solution, we prove that if $v_{t}$ is a classical solution of (1.1) satisfying $v_{0}=0$, then $v_{t}=0$ for all $t \geq 0$. In fact, since $v_{t}$ satisfies (1.1) and $v_{t} \in D\left(G_{m}\right)$, we have that

$$
B F v_{t}+\Phi v_{t}=\frac{d}{d t} F v_{t}=\lim _{h \rightarrow 0} \frac{F v_{t+h}-F v_{t}}{h}=F \lim _{h \rightarrow 0} \frac{v_{t+h}-v_{t}}{h}=F v_{t}^{\prime} .
$$

Therefore, $v_{t} \in D\left(G_{B, F, \Phi}\right)$ satisfies the Cauchy problem

$$
\begin{gathered}
\frac{d}{d t} v_{t}=G_{B, F, \Phi} v_{t} \quad \text { for } t \geq 0 \\
v_{0}=0
\end{gathered}
$$

Since $G_{B, F, \Phi}$ is the generator of a strongly continuous semigroup, this Cauchy problem has a unique solution $v_{t}=0$ (see [4, Theorem II.6.7]).

Finally, the last assertion, called the continuous dependence on the initial data of the solutions, follows from the uniform boundedness of the strongly continuous semigroup $\left(T_{B, F, \Phi}(t)\right)_{t \geq 0}$ on compact intervals.

3. Neutral semigroups with finite delay. In this section, we study (1.1) on a finite delay interval $[-r, 0]$, that is,

$$
\begin{aligned}
\frac{\partial}{\partial t} F u_{t} & =B F u_{t}+\Phi u_{t} \text { for } t \geq 0, \\
u_{0} & =\varphi \in C([-r, 0], X) .
\end{aligned}
$$


We again assume the difference operator $F$ to be given as in (2.7), that is,

$$
F \varphi=\varphi(0)-\Psi \varphi, \quad \varphi \in C([-r, 0], X)
$$

for some bounded linear operator $\Psi: C([-r, 0], X) \rightarrow X$. However, instead of assuming $\Psi$ to be "small," we suppose that $\Psi$ has no mass in 0 (see Definition 3.1). Our main idea is to renorm the space $C([-r, 0], X)$ such that, with the new equivalent norm, the norm of $\Psi$ is small, so we can adapt the arguments from the previous section. This idea has been used, for example, by Schwarz [11] to study ordinary neutral functional differential equations via dissipativity conditions. We begin with the definition of "no mass in 0. "

DEFINITION 3.1. A bounded linear operator $\Psi \in \mathscr{L}(C([-r, 0], X), X)$ is said to have no mass in 0 if for every $\epsilon>0$, there exists a positive number $\delta \leq r$ such that

$$
\|\Psi(f)\|_{X} \leq \epsilon\|f\|_{\infty} \quad \forall f \in C([-r, 0], X) \text { satisfying supp } f \subseteq[-\delta, 0] .
$$

REMARK 3.2. This definition is taken from [11, Definition II.2.1]. We note that, if $\Psi \in \mathscr{L}(C([-r, 0], X), X)$ has the form

$$
\Psi(f)=\int_{-r}^{0}[d \eta(\theta)] f(\theta)
$$

for some function $\eta(\cdot)$ of bounded variation, then the above definition is equivalent to the fact that the function $\eta(\cdot)$ is nonatomic at 0 in the sense of Hale and Verduyn Lunel [7, Chapter 9.2] or Wu [12, Chapter 2.3].

We are now prepared to renorm the space $C:=C([-r, 0], X)$. Indeed, for each positive number $\omega$, the new norm $\|\cdot\|_{\omega}$ defined by

$$
\|f\|_{\omega}:=\sup _{-r \leq s \leq 0}\left\|f(s) e^{-\omega s}\right\|_{X}, \quad f \in C
$$

is equivalent to the supnorm. Furthermore, $C_{\omega}$ denotes the space $C([-r, 0], X)$ endowed with the norm $\|\cdot\|_{\omega}$.

LEMMA 3.3. Let the operator $\Psi \in \mathscr{L}(C, X)$ have no mass in 0 . Then there exists a positive number $\omega$ such that the norm of the operator $\Psi$, as a bounded linear operator from $C_{\omega}$ into $X$, is smaller than 1 .

Proof. We first prove that there exists a number $\omega>0$ such that

$$
\|\Psi(f)\|_{X} \leq \frac{1}{2} \quad \forall f \in C([-r, 0], X) \text { satisfying }\|f(s)\| \leq e^{\omega s} \quad \forall s \in[-r, 0] .
$$

Indeed, since $\Psi$ has no mass in 0 , there exists a positive number $\delta \leq r$ such that $\|\Psi(f)\|_{X} \leq(1 / 8)\|f\|$ if supp $f \subseteq[-\delta, 0]$. For this $\delta$, we take an $\omega>0$ such that 
$\|\Psi\|<(1 / 4) e^{\delta \omega}$. Now, for a given $f \in C([-r, 0], X)$ satisfying $\|f(s)\|_{X} \leq e^{\omega s}$ for all $s \in[-r, 0]$, we prove that $\|\Psi(f)\|_{X} \leq 1 / 2$. For that purpose, we put

$$
f_{1}(s):= \begin{cases}f(s) & \text { for } s \in[-r,-\delta], \\ f(-\delta) & \text { otherwise, }\end{cases}
$$

and $f_{2}(s):=f(s)-f_{1}(s)$. Then, $\operatorname{supp} f_{2} \subseteq[-\delta, 0]$. Therefore, we have that

$$
\begin{aligned}
\|\Psi(f)\|_{X} & \leq\left\|\Psi\left(f_{1}\right)\right\|_{X}+\left\|\Psi\left(f_{2}\right)\right\|_{X} \\
& \leq\|\Psi\|\left\|f_{1}\right\|_{\infty}+\frac{1}{8}\left(\left\|f_{1}\right\|_{\infty}+\|f\|_{\infty}\right) \\
& <\frac{1}{2}
\end{aligned}
$$

and (3.6) follows.

Denote by $\|\Psi\|_{\omega}$ the norm of $\Psi$ as a bounded linear operator in $\mathscr{L}\left(C_{\omega}, X\right)$. Then, by inequality (3.6), we have that

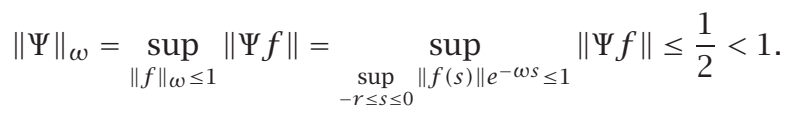

This renorming allows us to adapt the arguments from the proof of Theorem 2.4 to prove that the operator corresponding to (3.1) is the generator of a $C_{0^{-}}$semigroup on the space $C([-r, 0], X)$. Note that the generator property of an operator is preserved when passing to an equivalent norm.

THEOREM 3.4. Assume that the difference operator $F$ has the form $F \varphi=$ $\varphi(0)-\Psi \varphi$ with the bounded linear operator $\Psi: C([-r, 0], X) \rightarrow X$ having no mass in 0 , and let the operator $B$ generate a strongly continuous semigroup on $X$. Then, the operator $(G, D(G))$ defined by

$$
\begin{aligned}
G f & :=f^{\prime} \quad \text { on the domain } \\
D(G) & :=\left\{f \in C([-r, 0], X) \cap C^{1}([-r, 0], X): F f \in D(B), F f^{\prime}=B F f+\Phi f\right\}
\end{aligned}
$$

is the generator of a strongly continuous semigroup $(T(t))_{t \geq 0}$ on $C([-r, 0], X)$.

Proof. Let $C_{\omega}$ be the space $C([-r, 0], X)$ normed by the new norm $\|\cdot\|_{\omega}$ for $\omega$ as in Lemma 3.3. Then, the norm of the operator $\Psi$, as a bounded linear operator from $C_{\omega}$ into $X$, is smaller than 1. Therefore, as in Theorem 2.4, we show that the operator $(G, D(G))$ defined by (3.10) is densely defined and satisfies the Hille-Yosida estimates; hence, it generates a strongly continuous semigroup.

Analogously to Corollary 2.6, we have the following result about the existence, uniqueness, and continuous dependence on initial data of the solutions to the neutral PDE (3.1). 
COROLlary 3.5. Assume that the difference operator $F$ is of the form (3.2) such that $\Psi$ has no mass in 0 . Then, for every $\varphi \in D(G)$, there exists a unique classical solution $u_{t}(\cdot, \varphi)$ of (3.1), given by $u_{t}(\cdot, \varphi)=T(t) \varphi$, where the strongly continuous semigroup $(T(t))_{t \geq 0}$ is generated by the operator $G$ as in Theorem 3.4. Moreover, for every sequence $\left(\varphi_{n}\right)_{n \in \mathbb{N}} \subset D(G)$ satisfying $\lim _{n \rightarrow \infty} \varphi_{n}=0$, $\lim _{n \rightarrow \infty} u_{t}\left(\cdot, \varphi_{n}\right)=0$ uniformly in compact intervals.

Having established the well-posedness of (3.1), we now consider the robustness of the exponential stability of the solution semigroup. This can be done by using the constants appearing in the Hille-Yosida estimates of the operator $G$.

COROLLARY 3.6. Let the assumptions of Theorem 3.4 be satisfied. In addition, let the operator $B$ generate an exponentially stable $C_{0}$-semigroup and the norm of the operator $\Psi$ satisfy $\|\Psi\|<1$. Then, if the norm of the delay operator $\Phi$ is sufficiently small, the solution semigroup $(T(t))_{t \geq 0}$ generated by $(G, D(G))$ is exponentially stable.

Proof. We note that in the case of the finite delay interval $[-r, 0]$, the operators $e_{\lambda}$ defined as in (2.9) are well defined for all $\lambda \in \mathbb{C}$, and the exponent $\omega_{2}$ in the exponential estimate (2.5) can be chosen as $\omega_{2}:=\omega_{1}$ with the constant $M$ being replaced by $K:=\max \left\{M, M e^{-\omega_{1} r}\right\}$, where the constant $\omega_{1}$ appears in Assumption 1.1. Therefore, for (3.1) on the finite delay interval [ $-r, 0]$, we can adapt the arguments in the proof of Theorem 2.4 to obtain an analogue of Corollary 2.5, that is, the generator $(G, D(G))$ defined by (3.10) generates a strongly continuous semigroup $(T(t))_{t \geq 0}$ satisfying

$$
\|T(t)\| \leq P e^{\omega_{0} t}, \quad t \geq 0
$$

with the constant $P$ defined as in Theorem 2.4 and the constant

$$
\omega_{0}:=\max \left\{2 \omega_{1}+\frac{2 K\|\Phi\|}{1-\|\Psi\|}, \omega_{1}+\frac{K+K\|\Psi\|}{1-\|\Psi\|}\|\Phi\|\right\} .
$$

Now, the assumption that $\left(e^{t B}\right)_{t \geq 0}$ is exponentially stable means that $\omega_{1}<0$. Therefore, if $\|\Phi\|<-\omega_{1}(1-\|\Psi\|) /(K+K\|\Psi\|)$, then the solution semigroup is exponentially stable.

REMARK 3.7. In order to show the robustness of the exponential stability of the solution semigroup as in the above corollary, we need the condition $\|\Psi\|<1$. This is due to the fact that in the renorming Lemma 3.3, the constant $\omega>0$, which appears in the exponential bound of the solution semigroup, does not depend on the operator $B$. Moreover, we do not have an explicit estimate for this constant. This somehow corresponds to the fact considered by Hale [6, Theorem 1.2] that one needs some additional conditions on the difference operator $F$ to develop a qualitative theory of the neutral PDE (3.1). 
ACKNOWLEDGment. The second author gratefully acknowledges the support of the Vietnamese government.

\section{REFERENCES}

[1] M. Adimy and K. Ezzinbi, A class of linear partial neutral functional-differential equations with nondense domain, J. Differential Equations 147 (1998), no. 2, 285-332.

[2] R. Datko, Linear autonomous neutral differential equations in a Banach space, J. Differential Equations 25 (1977), no. 2, 258-274.

[3] K.-J. Engel, Spectral theory and generator property for one-sided coupled operator matrices, Semigroup Forum 58 (1999), no. 2, 267-295.

[4] K.-J. Engel and R. Nagel, One-Parameter Semigroups for Linear Evolution Equations, Graduate Texts in Mathematics, vol. 194, Springer-Verlag, New York, 2000.

[5] J. K. Hale, Coupled oscillators on a circle. Dynamical phase transitions (São Paulo, 1994), Resenhas 1 (1994), no. 4, 441-457.

[6] _ Partial neutral functional-differential equations, Rev. Roumaine Math. Pures Appl. 39 (1994), no. 4, 339-344.

[7] J. K. Hale and S. M. Verduyn Lunel, Introduction to Functional-Differential Equations, Applied Mathematical Sciences, vol. 99, Springer-Verlag, New York, 1993.

[8] N. T. Huy, Resolvents of operators and partial functional differential equations with non-autonomous past, Tübinger Berichte zur Funktionalanalysis $\mathbf{1 1}$ (2002), 239-252.

[9] F. Kappel and K. P. Zhang, Equivalence of functional-differential equations of neutral type and abstract Cauchy problems, Monatsh. Math. 101 (1986), no. 2, 115-133.

[10] _ On neutral functional-differential equations with nonatomic difference operator, J. Math. Anal. Appl. 113 (1986), no. 2, 311-343.

[11] M. Schwarz, Lineare Funktionaldifferentialgleichungen und ihre Lösungshalbgruppen, Ph.D. thesis, Universität Tübingen, Tübingen, 1989.

[12] J. Wu, Theory and Applications of Partial Functional-Differential Equations, Applied Mathematical Sciences, vol. 119, Springer-Verlag, New York, 1996.

[13] J. Wu and H. Xia, Self-sustained oscillations in a ring array of coupled lossless transmission lines, J. Differential Equations 124 (1996), no. 1, 247-278.

Rainer Nagel: Arbeitsgemeinschaft Funktionalanalysis (AGFA) Mathematisches Institut der Universität Tübingen, Auf der morgenstelle 10, 72076 Tübingen, Germany

E-mail address: rana@fa.uni-tuebingen.de

Nguyen Thieu Huy: Arbeitsgemeinschaft Funktionalanalysis (AGFA) Mathematisches Institut der Universität Tübingen, Auf der morgenstelle 10, 72076 Tübingen, Germany

E-mail address: hung@fa.uni-tuebingen.de 


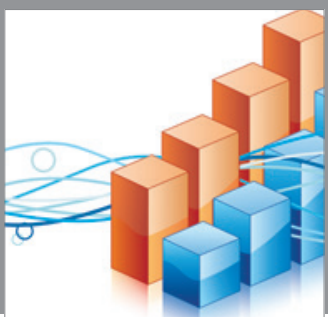

Advances in

Operations Research

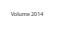

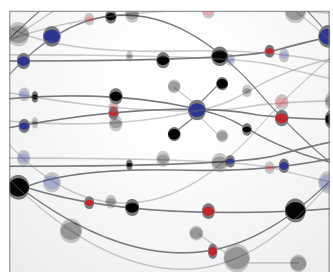

\section{The Scientific} World Journal
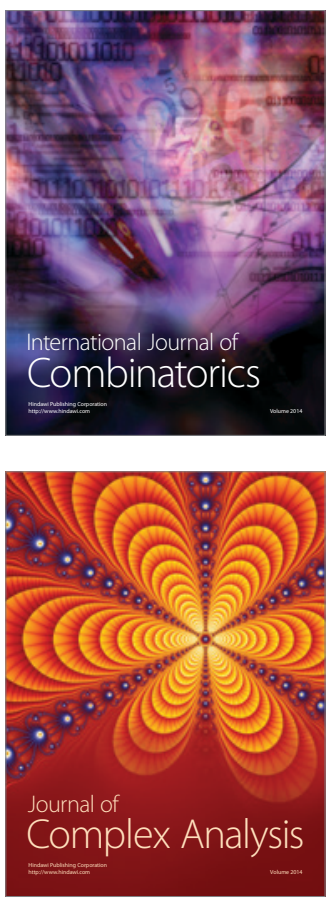

International Journal of

Mathematics and

Mathematical

Sciences
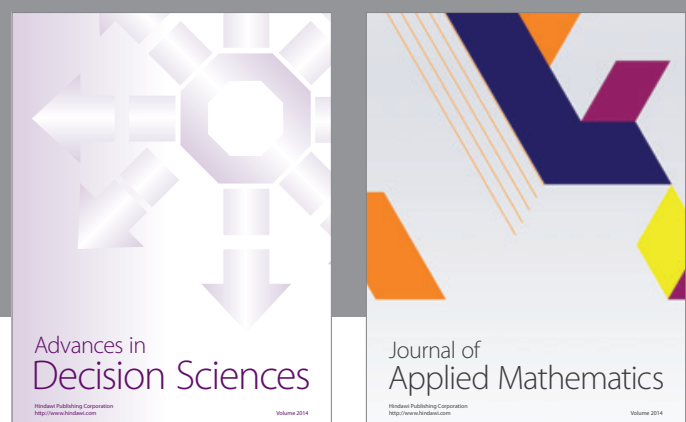

Journal of

Applied Mathematics
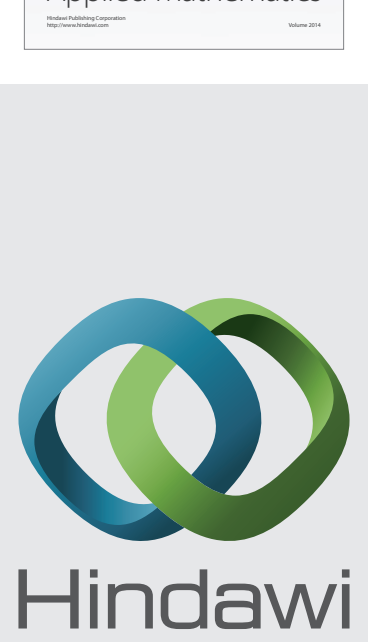

Submit your manuscripts at http://www.hindawi.com
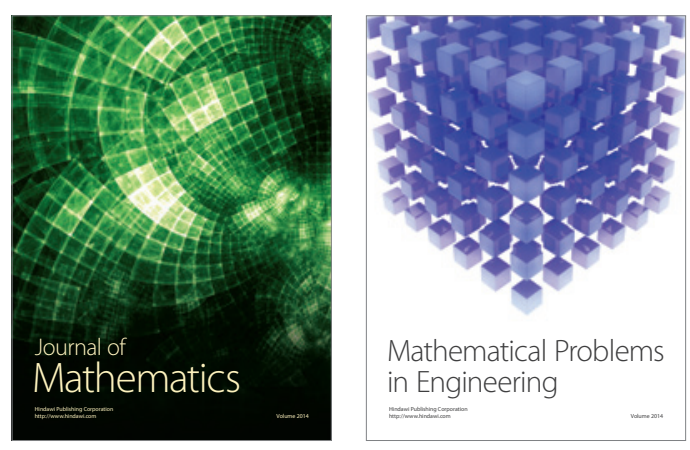

Mathematical Problems in Engineering
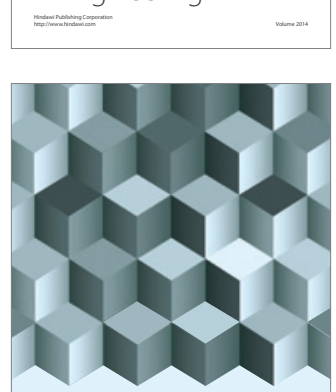

Journal of

Function Spaces
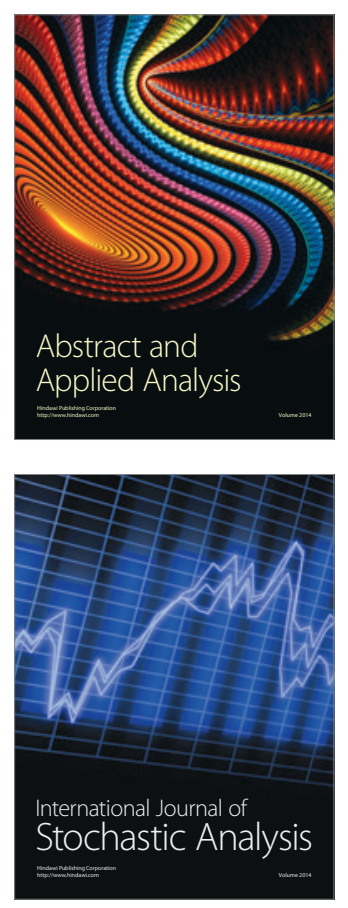

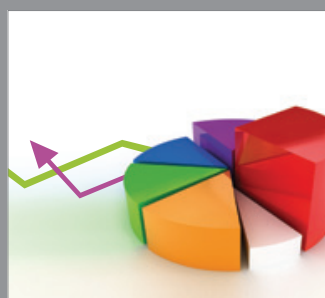

ournal of

Probability and Statistics

Promensencen
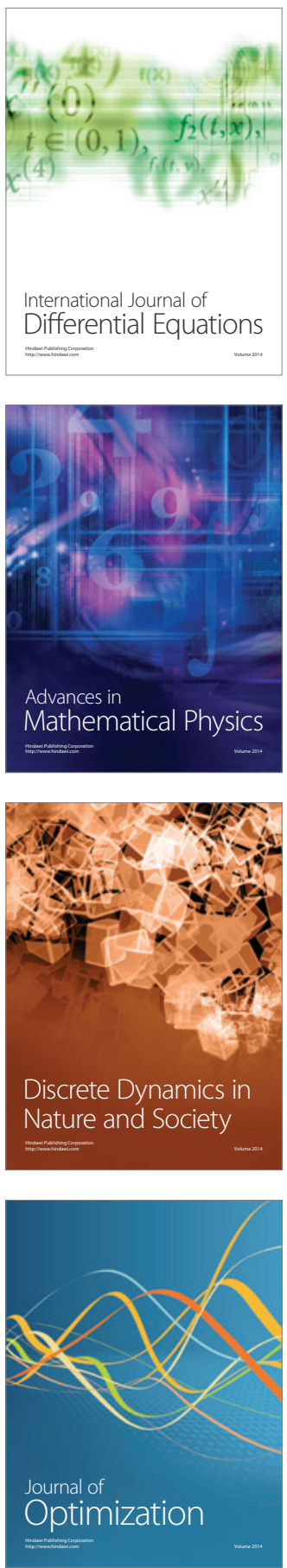